\title{
Analysis of T-Wave Alternans Using the Ramanujan Transform
}

\author{
LT Mainardi ${ }^{1}$, M Bertinelli $^{2}$, R Sassi $^{3}$ \\ ${ }^{1}$ Politecnico di Milano, Milano, Italy \\ ${ }^{2}$ et medical devices, Milano, Italy \\ ${ }^{3}$ Università di Milano, Crema, Italy
}

\begin{abstract}
In this study the use of Ramanujan transform (RT) for the assessment of T-Wave alternans (TWA) is investigated. The RT projects a signal on particular basis functions constituted by Ramanujan sums (RS). The resulting components highlight the presence of $1 / q$ resonances, being $q$ an integer number. Thus, the transform is suitable for detecting the typical $1 / 2$ pattern of TWA.

After alignment, the successive T-waves are filtered using the Amplitude Adjusted T-Average method. For each point of the obtained waveforms, the series of corresponding $T$ amplitudes is transformed using RT. Next, the quantification of TWA is obtained through the RT coefficients. Performances of the novel approach are evaluated on the Computers in Cardiology Challenge dataset 2008. The final score was encouraging (0.641), thus evidencing that $R T$ might be a valuable tool for the detection of TWA.
\end{abstract}

\section{Introduction}

T-Wave Alternans (TWA) is defined as a beat-to-beat alteration in the repolarisation morphology that repeats every other heart beat. The size of such alterations is small (about tens of microvolts) and they are often buried into noise. Therefore signal processing methods are most often necessary to enhance the signal and reveal their presence. A variety of methods have been proposed in the last two decades to automatically detect and estimate TWA in the ECG. A comprehensive and systematic discussion of methods for TWA detection and analysis is reported in [1].

In this paper we introduce a novel approach for TWA estimate based on the Ramanujan Transform (RT), which decomposes a signal as a combination of Ramanujan Sums (RS), each sum being characterized by sinusoids of integer periodicity $1 / q$ (with $q=\{1,2,3, \ldots, N\}$ and its multiples $p / q$ being $p$ and $q$ co-primes. The RT projects the original signal into patterns of finite periodicity: in the peculiar case of $q=2$, we have the 1 over 2 pattern typical of TWA. The RS have been introduced as the fundamental building blocks for arithmetical functions in number theory [2]. They have been recently rediscovered for signal processing [3] and for the analysis of biological data [4].

We investigated the performances of this novel approach in the estimation of TWA using the dataset of the Computers in Cardiology Challenge 2008.

\section{Methods}

\subsection{The Ramanujan transform}

Let's consider a $N$-samples signal, $x(n)$, and its decomposition as

$$
x(n)=\sum_{q=1}^{N} a_{q} c_{q}(n)
$$

where $n$ is the discrete-time index, the

$$
c_{q}(n)=\sum_{k=1 ;(k, q)=1}^{q} \exp \left(2 \pi j \frac{k}{q} n\right)
$$

is known as Ramanujan Sum (RS) and the $a_{q}$ 's are the Ramanujan coefficients. The symbolism $(k, q)$ which appears below the summation, denotes the greatest common divisor of $k$ and $q$ (thus the equality $(k, q)=1$ imposes $k$ and $q$ to be co-primes). One may interpret the RS as basis functions in which the original signal is projected: these basis functions satisfy many suitable properties for signal decomposition such as multiplicative and orthogonality properties.

It is interesting to observe the analogy between (1) and the discrete Fourier transform which is obtained by selecting

$$
c_{q}(n)=\exp \left(2 \pi j \frac{q}{N} n\right) .
$$

In (3) the basis functions are obtained by multiples of a fundamental frequency $1 / N$ defined by the signal length. In (2) the basis functions are obtained by summation of 
components which are multiples of the same integer periodicity $q$ being $q \in\{1,2,3,4, \ldots, \infty\}$. Thus the transformation is suitable to highlight the integer periodicity of the signal such as $1 / 2,1 / 3$ or $1 / N$ patterns, whose relevance is evaluated through the $a_{q}$ 's coefficients.

The computation of RS is obtained by

$$
c_{q}(n)=\mu\left(\frac{q}{(q, n)}\right) \frac{\phi(q)}{\phi\left(\frac{q}{(q, n)}\right)}
$$

where $\mu(\cdot)$ is the Moebius function and $\phi(\cdot)$ is the Euler totient function. Finally, the evaluation of Ramanujan coefficients is due to Charmichael [5]

$$
a_{q}(n)=\frac{1}{\phi(q)} \lim _{N \rightarrow \infty} \frac{1}{N} \sum_{n=1}^{N} x(n) c_{q}(n)
$$

\subsection{Database}

The database employed to assess the performances of our method is the one provided for the PhysioNet/Computers in Cardiology Challenge 2008. It contains 100 multichannel ECG records sampled at $500 \mathrm{~Hz}$ with 16 bits resolution over a $\pm 32 \mathrm{mV}$ range. The dataset includes patients with myocardial infarctions, transient ischemia, ventricular tachyarrhythmias, and other risk factors for sudden cardiac death, as well as healthy controls. It also contains 32 synthetic records obtained with 6 different models. The challenge's participants were not aware of which records were real or synthetic; also if TWA was present or not in a specific subject was undisclosed. For further details on the dataset please refer to [6].

\subsection{Signal pre-processing}

The dataset did not contain annotations. Thus, each record was firstly processed for QRS detection and classification using a refined version of the freely available software OSEA [7]. The ECG signals were first highpass filtered to remove major baseline wander (3th order Butterworth filter, cut off frequency $0.5 \mathrm{~Hz}$ ). Then, each lead was analyzed separately, for Q onsets detection. Fiducial points obtained in different leads were merged if closer than $150 \mathrm{~ms}$. OSEA furnished a rough classification of each beat. To refine it further and exclude remaining ventricular beats, artifacts and labeling errors, we marked as artifacts those beats which showed a maximal crosscorrelation value $<0.9$ with an average template. The cross-correlation was computed with a shift of up to \pm 100 $\mathrm{ms}$, and the Q onsets positions were relocated accordingly. The process was repeated iteratively until no change was detected or a maximum number of iteration was reached. We finally averaged normal beats and used this final template to detect waves boundaries (QRS and T offsets; T onsets were defined as QRS offsets + $40 \mathrm{~ms}$ ).

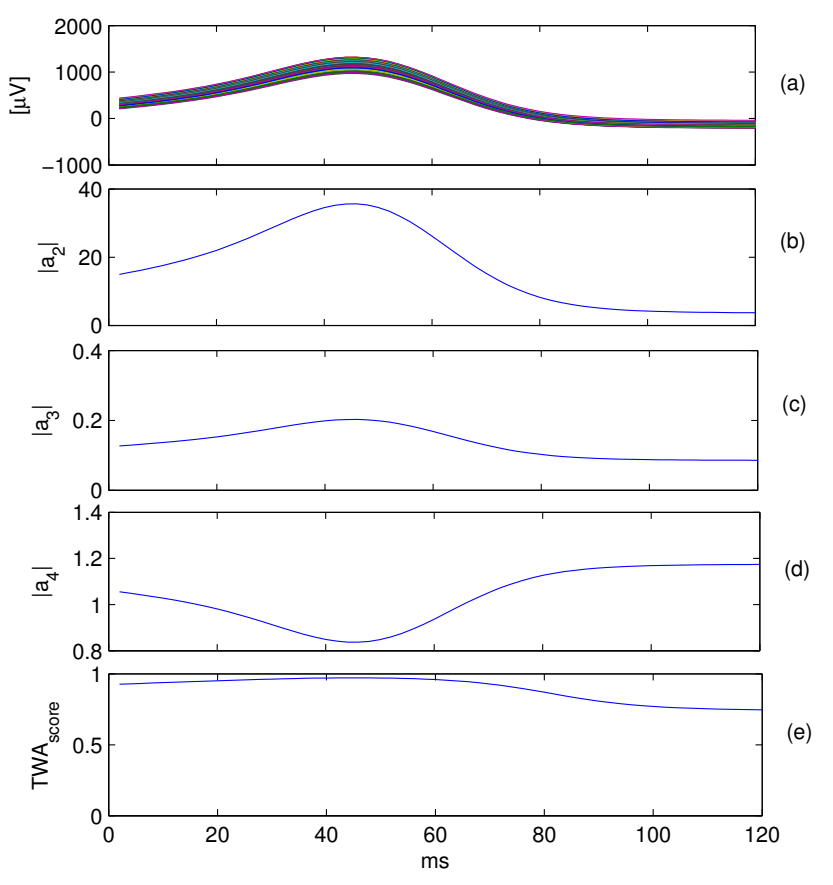

Figure 1. Computation of $T W A_{\text {score }}$ on lead V4 of record 79. a) Superimposition of filtered T-waves using the AATA method; b)-d) modulus of Ramanujan coefficients for $q=2,3,4$ and e) the resulting $T W A_{\text {score }}$. Note that the score has an high value during the entire $\mathrm{T}$ duration as expected in case of TWA.

Finally, a template for the $\mathrm{T}$ wave was built and used to refine through cross-correlation the position of the $\mathrm{T}$ onsets $( \pm 100 \mathrm{~ms})$. Also in this case the process was repeated iteratively. The temporal alignment of the $\mathrm{T}$ waves is at the core of how we employed the Ramanujan transform. The alignment procedure generates a series of aligned $\mathrm{T}$ waves, $T_{k}^{i}(n) k=\{1,2, \ldots, N\}$ (being $N$ the number of $\mathrm{T}$ waves in the record), and a series of cross-correlation values $C_{k}^{i}$ between each T-wave and the template. Let's call $T_{\text {ave }}^{i}(n)$ the average $\mathrm{T}$ complex computed on the $i$-th lead, being $n$ the discrete time index ( $n=0$ corresponds to the $\mathrm{T}$ onset). The average correlation was also computed for each available lead $C_{a}^{i}=E\left[C_{k}^{i}\right]$.

\subsection{T-Wave alternans quantification}

The first step was the selection of the T-waves to be included in the TWA computation. This was done through the joint analysis of RR intervals and $C_{k}^{i}$ values. In details, we selected the longest sequence in which: i) two successive RR intervals did not differ more than a $10 \%$ in respect to the mean global RR value; and ii) all the $C_{k}^{i}$ were greater than 0.9 . These criteria guaranteed to generate a sequence of similar $\mathrm{T}$ waves, with no $\mathrm{T}$-wave missed (or incorrectly added). 
To reduce the influence of noise in the estimation of TWA amplitude, we designed the Amplitude Adjusted TAverage (AATA) method, in which the aligned $T_{k}^{i}(n)$ 's were modeled as amplitude-adjusted version of the Taverage:

$$
T_{k}^{i}(n)=c_{k} T_{a v e}^{i}(n)+d_{k}+w(n)=A A T_{k}^{i}(n)+w(n)
$$

where $w(n)$ is the additive noise term. For each T-wave, the values of coefficients $c_{k}$ and $d_{k}$ are estimated in the least square sense. The successive analysis was performed on the sequence of $A A T_{k}^{i}(n)$ instead of the original $T_{k}^{i}(n)$.

For each time instant $n$, the series of corresponding $A A T_{k}^{i}(n)$ amplitudes was transformed using the RT; the modulus of the RT coefficients $\left|a_{q}^{i}(n)\right|$ derived for $q=$ $2,3,4$ were further processed. It is worth noting that $\left|a_{2}^{i}(n)\right|$ is an estimate of the TWA, while coefficients $\left|a_{3}^{i}(n)\right|$ and $\left|a_{4}^{i}(n)\right|$ are estimators of the superimposed noise level. These coefficients were derived for each point $n$ of the $\mathrm{T}$ wave and for each lead $i$.

To measure the prevalence of $1 / 2$ pattern in the series, the following score was computed using the RT coefficients

$$
T W A_{\text {score }}^{i}(n)=\frac{\left|a_{2}^{i}(n)\right|}{\left|a_{2}^{i}(n)\right|+\left|a_{3}^{i}(n)\right|+\left|a_{4}^{i}(n)\right|}
$$

The score tends to 1 when the $1 / 2$ pattern is dominant, i.e. $\left|a_{2}^{i}(n)\right|>>\left|a_{3}^{i}(n)\right|+\left|a_{4}^{i}(n)\right|$. Conversely, it goes to 0 when the $1 / 2$ pattern is negligible.

The amplitude of TWA was computed in a temporal window $( \pm 16 \mathrm{~ms})$ centered around the T-peak. In this interval, the maximal values of $2\left|a_{2}^{i}(n)\right|$ was selected as estimator of TWA amplitude. However, if the $T W A_{\text {score }}^{i}$ was lower than 0.3 in correspondence of this maximum, the measure was considered unreliable and TWA amplitude was set to 0 . This rule was included to avoid false TWA detections in noisy ECG.

\subsection{Merging information from different leads}

The computations described in the previous section were repeated separately for the available leads (2,3 or 12 depending on the record). However, the Challenge required to provide a single score for each record. Therefore information coming from different leads were merged to define the final TWA estimates.

Noisy leads did not entered the merging process. A lead was defined as noisy if $C_{a}^{i}<0.8$ (see definition of $C_{a}^{i}$ in section 2.3) and excluded. Among the remaining ones, those having $T W A_{\text {score }}^{i}>0.8$ were selected and the maximum of $2\left|a_{2}^{i}\right|$ was elected as TWA estimator. If none of the leads satisfied the last condition, the lead with the largest $T W A_{\text {score }}^{i}$ was selected and the corresponding $2\left|a_{2}^{i}\right|$ used to measure TWA.

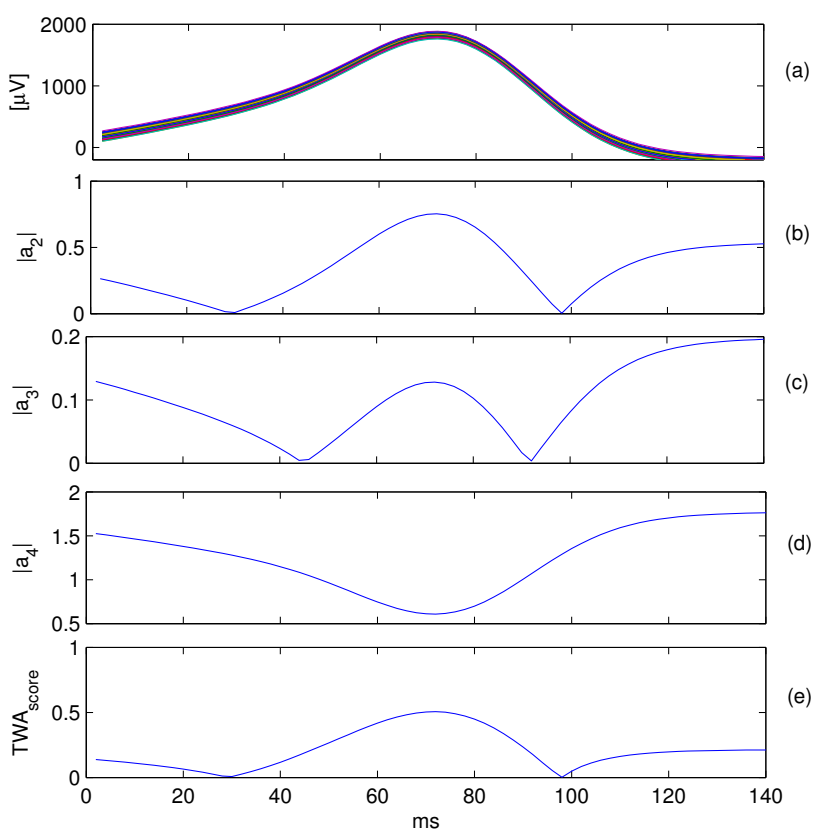

Figure 2. Computation of $T W A_{\text {score }}$ on lead V4 of record 52. a) Superimposition of filtered T-waves using the AATA method; b)-d) modulus of Ramanujan coefficients for $q=2,3,4$ and e) the resulting $T W A_{\text {score }}$. The score is low during the entire $\mathrm{T}$ duration. The probability of TWA being present is low.

\section{Results}

Comparison of results obtained in presence/absence of TWA are shown in Figure 1 and Figure 2, respectively. Both the Ramanujan coefficient $\left|a_{2}\right|$ and the $T W A_{\text {score }}$ decrease when TWA is not present. We observed this behavior to be typical in the database.

During the Challenge we submitted 4 valid entries. The best performance was obtained at the fourth submission: our final score was 0.641 in the range $[-1,1]$.

After the end of the Challenge, the list of 32 records with artificially generated TWA were published. The synthetic record were generated using different ECG models and superimposing various TWA levels, which were also disclosed.

The comparison between reference values and our estimates allowed a finer tuning of the algorithm parameters. In particular, the threshold on $T W A_{\text {score }}^{i}$ (see section 2.5) was decreased in order to include more leads in the selection of the final TWA estimates. This was in favor of selecting the same lead when different realizations of the same ECG model were considered.

The results obtained on the synthetic records are shown in Figure 3. We observed that our performances were model dependent. In particular, in model A, B and E our estimates were nearly correct (the slope of the line fitting 


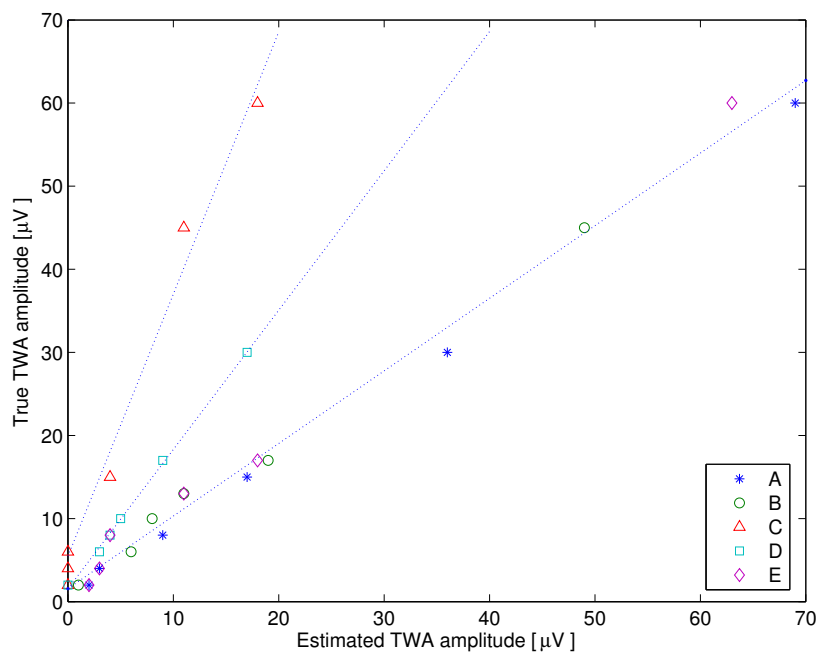

Figure 3. Comparison between estimated and true TWA amplitude on the records of model A,B,C,D and E

was 0.874 ), while in model $\mathrm{C}$ and $\mathrm{D}$ we under-estimated the true TWA amplitudes. Interestingly, the level of this underestimation was constant with ECGs coming from the same model: in model $\mathrm{E}$, the estimations were roughly $1 / 2$ of the true values; in model $\mathrm{C}$, they were $1 / 3$ of the true ones.

Generally, we were able to determine a correct ranking of the members of each group, but the systematic underestimation in model $\mathrm{C}$ and $\mathrm{D}$ reduced the score on the overall ranking. In addition, the underestimation in model $\mathrm{C}$ prevents, for this group, the detection of TWA events with amplitude $<6 \mu V$.

\section{Discussion and conclusions}

In this work we derived a TWA detector based on the Ramanujan transform. The peculiar property of this transformation allows the detection of $1 / 2,1 / 3,1 / 4$ patterns in the signal and it is therefore suitable for TWA detection. In fact, the prevalence of $1 / 2$ rhythms over longer $1 / 3,1 / 4, \ldots, 1 / N$ periodicity may be easily inferred from the analysis of the Ramanujan coefficients, which we used to derive an index of TWA events. In addition, it can be shown that RS are constituted by integer number. Therefore, computation of Ramanujan coefficients (equation 5) is rather fast, efficient and easy-to-implement.

As expected, the output of RT may be affected by ECG noise. Therefore, we design a pre-processing procedure to reduce noise artifact and derive a robust TWA estimator. T-waves were firstly aligned, and then filtered using AATA method, in which the T's were modeled as amplitude- adjusted version of the gross $\mathrm{T}$-average. This procedure drastically reduces the superimposed noise and improve the results.

A major problem of our approach was related to the need for synthesizing the information coming from different leads. In the Challenge, our philosophy was to estimate the TWA amplitude as the maximum TWA estimates among all the leads. However, from the analysis of synthetic ECGs, a different strategy, which privileges the selection of the same lead among groups, seems more profitable. Unfortunately, we are not able to verify if this change of strategy would have also improved the Challenge score on the real part of the database.

In conclusions, this work shows the potentiality of the Ramanujan transform for the detection and the quantitative evaluation of TWA episodes. Even if further improvements are still needed to enhance the performances of the method, the score we obtained is encouraging. Once the information on the true ranking of the database will be available a further improvement is expected.

\section{References}

[1] Martínez JP, Olmos S. Methodological principles of T wave alternans analysis: a unified framework. IEEE Trans Biomed Eng Apr 2005;52(4):599-613.

[2] Ramanujan S. On certain trigonometrical sums and their application in the theory of numbers. Trans Camb Phil Soc 1918;22:259-276.

[3] Planat M, Rosu H, Perrine S. Ramanujan sums for signal processing of low-frequency noise. Phys Rev E Stat Nonlin Soft Matter Phys Nov 2002;66(5 Pt 2):056128.

[4] Mainardi LT, Pattini L, Cerutti S. Application of the Ramanujan Fourier Transform for the analysis of secondary structure content in amino acid sequences. Methods Inf Med 2007;46(2):126-129.

[5] Carmichael R. Exapnsions of arthmetical functions in infinite series. Proc London Math Soc 1932;34:2.

[6] Moody G. The physionet / Computers in Cardiology Challenge 2008: T-Wave Alternans. In Proc. Computers in Cardiology. 2008; .

[7] Hamilton P. Open source ECG analysis software. Available from www. eplimited.com.

Address for correspondence:

Luca Mainardi

Dipartimento di Bioingegneria

Politecnico di Milano

Piazza Leoanrdo da Vinci 32, 20133 Milano (MI), Italy

E-mail address: luca.mainardi@biomed.polimi.it 